\title{
Porphyrin-Containing Polymacrocycles: Synthesis and Evaluation as Fluorescent Detectors of Metal Cations
}

\author{
Alexei A. Yakushev, ${ }^{\text {a }}$ Alexei D. Averin, ${ }^{\mathrm{a}, \mathrm{b} @}$ Olga A. Maloshitskaya, ${ }^{\mathrm{b}}$ Oskar I. Koifman, ${ }^{\mathrm{c}}$ \\ Sergei A. Syrbu, ${ }^{\mathrm{d}}$ and Irina P. Beletskaya ${ }^{\mathrm{a}, \mathrm{b}}$ \\ ${ }^{a}$ A.N. Frumkin Institute of Physical Chemistry and Electrochemistry RAS, 119991 Moscow, Russia \\ ${ }^{\mathrm{b}}$ Lomonosov Moscow State University, Department of Chemistry, 119991 Moscow, Russia \\ 'Ivanovo State University of Chemistry and Technology, 153000 Ivanovo, Russia \\ ${ }^{\mathrm{d}}$ G.A. Krestov Institute of Solution Chemistry RAS, 153045 Ivanovo, Russia \\ ${ }^{\circledR}$ Corresponding author E-mail: alexaveron@yandex.ru
}

\begin{abstract}
Using Pd(0)-catalyzed amination reaction of zinc 5,15-bis(4-bromophenyl)porphyrinate with diazatrioxamacrocycle-derivative of 3,3'-disubstituted biphenyl-a series of polymacrocyclic compounds was obtained. The investigation of their fluorescence in the presence of 18 metal cations revealed that two of them can act as molecular probes for Cu(II), Al(III) and Cr(III) by the fluorescence quenching. Tri-and tetramacrocyclic compounds of another structure were synthesized by the Pd(0)-catalyzed arylation of cryptands comprising central diazacrown ether moieties using zinc 5-(4-bromophenyl)porphyrinate. One of these compounds was characterized as a fluorescent chemosensor for $\mathrm{Cu}(I I)$.
\end{abstract}

Keywords: Porphyrins, diazacrown ethers, polymacrocycles, Pd catalysis, amination, fluorescence, detection.

\section{Порфирин-содержащие полимакроциклы: синтез и оценка в качестве флуоресцентных детекторов катионов металлов}

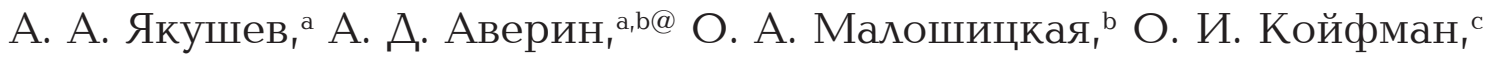 \\ С. А. Сырбу, ${ }^{d}$ И. П. Белецкая ${ }^{\mathrm{a}, \mathrm{b}}$ \\ ${ }^{a}$ Институт физической химии и электрохимии им. А.Н. Фрумкина РАН, 119991 Москва, Россия \\ ${ }^{\mathrm{b}}$ Московский государственный университет им. М.В. Ломоносова, Химический факультет, 119991 Москва, Россия \\ ${ }^{\mathrm{c}}$ Ивановский государственный химико-технологический университет, 153000 Иваново, Россия \\ ${ }^{\mathrm{d}}$ Институт химии растворов им. Г.А. Крестова РАН, 153045 Иваново, Россия \\ @E-mail: alexaveron@yandex.ru
}

С использованием Pd(0)-катализируемой реакции аминирования цинкового комплекса 5,15-бис(4-бромфенил) порфирина с диазатриоксамакроциклом - производным 3,3'-дизамещенного бифенила - получена серия полимакроциклических соединений. Исследование их флуоресценции в присутствии катионов 18 металлов показало, что два из них могут выступать в качестве молекулярных проб на катионы Сu(II), Al(III) и Cr(III) за счет тушения флуоресценции. Три- и тетрамакрочиклические соединения другого строения синтезировань Pd(0)-катализируемылм аминированием криптандов, содержащчих иенттральный фрагмент диазакраун-эфира с использованием ичинкового комплекса 5-(4-бромфенил)порфирина. Одно из этих соединений охарактеризовано как флуоресиентный хемосенсор на катионы Си(II).

Ключевые слова: Порфирины, диазакраун-эфиры, полимакроциклы, Pd катализ, аминирование, флуоресценция, детектирование. 


\section{Introduction}

Catalytic approaches to polymacrocyclic compounds based on porphyrins are well documented. The vast majority of such molecules are porphyrin dyads, triads and parent compounds, while the conjugates with other nitrogencontaining macrocycles are still enough rare. The synthesis of directly meso-meso-linked porphyrins without any spacer can be achieved via $\mathrm{Ni}$ (II)-catalyzed oxidative coupling of meso-bromoporphyrins ${ }^{[1]}$ or using Suzuki coupling. ${ }^{[2,3]}$ The chemistry of porphyrin oligomers built using various aromatic, heteroaromatic or other unsaturated linkers is much more explored as they can bring additional structural and physicochemical properties to a molecule. For this purpose Suzuki, ${ }^{[4-6]}$ Stille, ${ }^{[7,8]}$ Heck, ${ }^{[9]}$ and Sonogashira ${ }^{[10,11]}$ couplings were successfully applied. Triazolyl linker can be easily introduced in the porphyrin dyads and triads by epy so-called click reactions, ${ }^{[12-14]}$ and the application of Buchwald-Hartwig amination reactions was reported for the synthesis of bisporphyrin compounds in which two macroheterocyles were linked with a simple NH fragment, ${ }^{[15]}$ a series of di- an polyporphyrin compounds was obtained by a similar approach in which diamines or diazacrown ether moieties served as linkers. ${ }^{[16,17]}$ While porphyrins possess extremely interesting photophysical properties together with their unique binding of metal cations, they have not yet become a widespread platform for creating colorimetric or fluorimetric chemosensors. The examples are still scarce, to mention the porphyrin-terpyridine conjugate which can detect $\mathrm{Cd}(\mathrm{II})$ with moderate selectivity, ${ }^{[18]}$ tetrakis(4methoxyphenyl)porphyrin for sensing $\operatorname{Ag}(\mathrm{I}),{ }^{[19]}$ and another detector of the same cation employing the combination of the porphyrin and quinoline moieties. ${ }^{[20]}$ Recently we have described the catalytic synthesis of porphyrin conjugates with azacrown ethers and tested them as fluorimetric detectors of metal cations. ${ }^{[21,22]}$ In continuation of this research here we report the synthesis of the polymacrocyclic derivatives of porphyrin comprising biphenyl-based diazatrioxamacrocycle and cryptands with diazacrown ether moieties.

\section{Experimental}

NMR spectra were registered using Bruker Avance 400 spectrometer, MALDI-TOF spectra were obtained with Bruker Autoflex II spectrometer using 1,8,9-trihydroxyanthracene as matrix and PEGs as internal standards. UV-Vis spectra were recorded with Agilent Cary 60 spectrophotometer in $\mathrm{MeCN}$, spectra of fluorescence were obtained with Hitachi 2700 spectrofluorometer in acetonitrile (UHPLC grade). Rac-BINAP and DavePhos ligands, sodium tert-butoxide, were purchased from Sigma-Aldrich Co and used without further purification, $\mathrm{Pd}(\mathrm{dba})_{2}$ was synthesized according to the method described. ${ }^{[23]}$ Macrobicycle 3 was obtained according to the described method, ${ }^{[24]}$ macrobicycles 12-14 were synthesized according to a published procedure, ${ }^{[25]}$ zinc porphyrinates 4,15 and 18 were obtained by method described in ref. ${ }^{[26]}$ Column chromatography was carried out using silica gel 40-63 nm (Fluka). Acetonitrile of UHPLC grade was used without additional purification, dioxane was successively distilled over $\mathrm{NaOH}$ and sodium. Dichloromethane was distilled over $\mathrm{CaH}_{2}$, methanol was used freshly distilled.

Method for the synthesis of polymacrocycles 5-8, 16, 17, 19, 20. A two-neck flask equipped with a magnetic stirrer and reflux condenser, flushed with dry argon, was charged with corresponding amounts of $\operatorname{Pd}(\mathrm{dba})_{2}$, DavePhos, zinc porphyrinates 4 or 18, macrocyclic compound 3 or cryptands 12-14, absolute dioxane and sodium tert-butoxide. The reaction mixture was stirred under reflux for $24 \mathrm{~h}$, cooled down to ambient temperature, the residue was filtered off, washed with $\mathrm{CH}_{2} \mathrm{Cl}_{2}(5 \mathrm{ml})$, the combined organic fractions were evaporated in vacuo and the residue was chromatographed on silica gel using a sequence of eluents: $\mathrm{CH}_{2} \mathrm{Cl}_{2}, \mathrm{CH}_{2} \mathrm{Cl}_{2}$ - $\mathrm{MeOH}$ 500:1 - 3:1.

Trismacrocyclic compound 5. Obtained from zinc porphyrinate $4(0.1 \mathrm{mmol}, 102 \mathrm{mg})$, macrocycle $3(0.2 \mathrm{mmol}, 74 \mathrm{mg})$ in the presence of Pd(dba) $(16 \mathrm{~mol} \%, 9 \mathrm{mg})$, DavePhos (18 mol \%, $7 \mathrm{mg}), t \mathrm{BuONa}(0.3 \mathrm{mmol}, 29 \mathrm{mg})$ in $2 \mathrm{ml}$ dioxane. Eluent: $\mathrm{CH}_{2} \mathrm{Cl}_{2}-\mathrm{MeOH}$ 100:1. Yield $14 \mathrm{mg}$ (9\%), dark-red crystalline powder. M.p. $195-200{ }^{\circ} \mathrm{C} . \mathrm{m} / z$ (MALDI-TOF) found: 1596.9069 . $\mathrm{C}_{100} \mathrm{H}_{124} \mathrm{~N}_{8} \mathrm{O}_{6} \mathrm{Zn}$ requires $1596.8935[\mathrm{M}]^{+}$. UV-Vis $\left(\mathrm{CH}_{3} \mathrm{CN}\right) \lambda_{\text {n }}$ (lge) nm: 416 (5.29). ${ }^{1} \mathrm{H}$ NMR $\left(\mathrm{CDCl}_{3}, 298 \mathrm{~K}\right) \delta_{\mathrm{H}} \mathrm{ppm}: 1.00 \mathrm{t}$ $\left(12 \mathrm{H},{ }^{3} J=7.3 \mathrm{~Hz}\right), 1.54-1.61 \mathrm{~m}(12 \mathrm{H}), 1.78$ quintet $\left(8 \mathrm{H},{ }^{3} J=7.4 \mathrm{~Hz}\right)$, $2.17-2.26 \mathrm{~m}(12 \mathrm{H}), 2.63 \mathrm{~s}(12 \mathrm{H}), 3.24$ br.t $\left(4 \mathrm{H},{ }^{3} \mathrm{~J}_{o b s}=4.7 \mathrm{~Hz}\right), 3.31$ br.s $(4 \mathrm{H}), 3.33-3.63 \mathrm{~m}(16 \mathrm{H}), 3.67$ br.t $\left(4 \mathrm{H},{ }^{3} J=4.9 \mathrm{~Hz}\right), 4.00$ br.s $(8 \mathrm{H}), 4.21 \mathrm{t}\left(4 \mathrm{H},{ }^{3} J=6.1 \mathrm{~Hz}\right), 5.70$ br.s $(2 \mathrm{H}), 6.12$ br.s $(2 \mathrm{H}), 6.90 \mathrm{~d}$ $\left(2 \mathrm{H},{ }^{3} J=7.2 \mathrm{~Hz}\right), 7.00 \mathrm{t}\left(2 \mathrm{H},{ }^{3} J=7.1 \mathrm{~Hz}\right), 7.16 \mathrm{~d}\left(2 \mathrm{H},{ }^{3} J=7.3 \mathrm{~Hz}\right), 7.28$ $\mathrm{d}\left(2 \mathrm{H},{ }^{3} J=8.5 \mathrm{~Hz}\right), 7.36-7.43 \mathrm{~m}(8 \mathrm{H}), 7.87 \mathrm{~d}\left(4 \mathrm{H},{ }^{3} J=7.6 \mathrm{~Hz}\right), 10.14$ $\mathrm{s}(2 \mathrm{H})$ (NH protons were not assigned). ${ }^{13} \mathrm{C} \mathrm{NMR}\left(\mathrm{CDCl}_{3}, 298 \mathrm{~K}\right)$

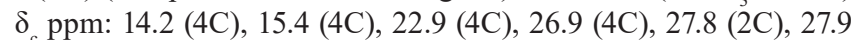
(2C), 32.7 (4C), 33.2 (4C), 42.9 (2C), 49.1 (2C), 68.5 (2C), 70.5 (2C), 70.7 (2C), 70.8 (2C), 70.9 (2C), 80.0 (2C), 97.2 (2C), 111.2 (2C), 113.1 (2C), 116.9 (2C), 118.9 (2C), 120.2 (4C), 120.4 (2C), $129.0,129.1,134.1$ (4C), 137.2, 137.9, 141.9, 143.1, 146.3, 147.2, $147.5,148.1,149.3$ (6 quaternary carbon atoms of the biphenyl moieties were not assigned, carbon atoms of the porphyrin moiety were not integrated).

Bismacrocycle 6 was isolated as the second compound in the synthesis of trismacrocycle 5. Eluent: $\mathrm{CH}_{2} \mathrm{Cl}_{2}-\mathrm{MeOH} 500: 1$. Yield $20 \mathrm{mg}$ (16\%), dark-red crystalline powder. M.p. 148-150 ${ }^{\circ} \mathrm{C} . \mathrm{m} / z$ (MALDI-TOF) found: $1228.6770 . \mathrm{C}_{78} \mathrm{H}_{96} \mathrm{~N}_{6} \mathrm{O}_{3} \mathrm{Zn}$ requires $1228.6835[\mathrm{M}]^{+}$. UV-Vis $\left(\mathrm{CH}_{3} \mathrm{CN}\right) \lambda_{\text {max }}(\lg \varepsilon) \mathrm{nm}: 414$ (5.28). ${ }^{1} \mathrm{H}$ NMR $\left(\mathrm{CDCl}_{3}, 298 \mathrm{~K}\right) \delta_{\mathrm{H}}$ ppm: $0.98 \mathrm{t}\left(6 \mathrm{H},{ }^{3} J=7.1 \mathrm{~Hz}\right), 0.99 \mathrm{t}(6 \mathrm{H}$, $\left.{ }^{3} J=7.1 \mathrm{~Hz}\right), 1.52-1.60 \mathrm{~m}(10 \mathrm{H}), 1.70-1.80 \mathrm{~m}(8 \mathrm{H}), 2.16-2.25 \mathrm{~m}$ $(10 \mathrm{H}), 2.45 \mathrm{~s}(6 \mathrm{H}), 2.61 \mathrm{~s}(6 \mathrm{H}), 3.13$ br.t $\left(2 \mathrm{H},{ }^{3} \mathrm{~J}=4.5 \mathrm{~Hz}\right), 3.23$ br.s $(2 \mathrm{H}), 3.33-3.60 \mathrm{~m}(8 \mathrm{H}), 3.65$ br.t $\left(2 \mathrm{H},{ }^{3} J_{o b s}=5.3 \mathrm{~Hz}\right), 3.93-4.02$ $\mathrm{m}(8 \mathrm{H}), 4.20 \mathrm{t}\left(2 \mathrm{H},{ }^{3} \mathrm{~J}=6.1 \mathrm{~Hz}\right), 5.49$ br.s $(1 \mathrm{H}), 5.91$ br.s $(1 \mathrm{H}), 6.87$ d $\left(1 \mathrm{H},{ }^{3} J=7.3 \mathrm{~Hz}\right), 6.93 \mathrm{t}\left(1 \mathrm{H},{ }^{3} J=7.3 \mathrm{~Hz}\right), 7.14 \mathrm{~d}\left(1 \mathrm{H},{ }^{3} J=7.2 \mathrm{~Hz}\right)$, $\left.7.27 \mathrm{~d}\left(1 \mathrm{H},{ }^{3} \mathrm{~J}=8.7 \mathrm{~Hz}\right), 7.31-7.40 \mathrm{~m}(5 \mathrm{H}), 7.70-7.80 \mathrm{~m} \mathrm{(2H}\right), 7.84 \mathrm{~d}$ $\left(2 \mathrm{H},{ }^{3} \mathrm{~J}=8.0 \mathrm{~Hz}\right), 8.08 \mathrm{~d}\left(2 \mathrm{H},{ }^{3} \mathrm{~J}=8.0 \mathrm{~Hz}\right), 10.14 \mathrm{~s}(2 \mathrm{H})(\mathrm{NH}$ protons were not assigned).

Tetramacrocycle 7 was isolated as the third compound in the synthesis of trismacrocycle 5. Eluent: $\mathrm{CH}_{2} \mathrm{Cl}_{2}-\mathrm{MeOH}$ 200:1. Yield $11 \mathrm{mg}(9 \%)$, dark-red solid. $\mathrm{m} / z$ (MALDI-TOF) found: 2455.42. $\mathrm{C}_{156} \mathrm{H}_{190} \mathrm{~N}_{12} \mathrm{O}_{6} \mathrm{Zn}_{2}$ requires $2455.35[\mathrm{M}]^{+}$. ${ }^{1} \mathrm{H} \mathrm{NMR}\left(\mathrm{CDCl}_{3}\right.$, $298 \mathrm{~K}) \delta_{\mathrm{H}}$ ppm: $0.96 \mathrm{t}\left(6 \mathrm{H},{ }^{3} J=7.1 \mathrm{~Hz}\right), 0.98 \mathrm{t}\left(18 \mathrm{H},{ }^{3} \mathrm{~J}=6.9 \mathrm{~Hz}\right), 1.56$ br.s (16H), 1.75 br.s (16H), 1.84-1.94 m (6H), 2.20 br.s (18H), $2.63 \mathrm{~s}$ $(12 \mathrm{H}), 2.67 \mathrm{~s}(12 \mathrm{H}), 3.22 \mathrm{t}\left(2 \mathrm{H},{ }^{3} \mathrm{~J}=5.6 \mathrm{~Hz}\right), 3.40-3.78 \mathrm{~m}(24 \mathrm{H}), 3.92$ br.s $(4 \mathrm{H}), 3.99$ br.s $(12 \mathrm{H}), 4.22$ br.s $(6 \mathrm{H}), 6.50 \mathrm{~d}\left(2 \mathrm{H},{ }^{3} \mathrm{~J}=7.8 \mathrm{~Hz}\right)$, $6.60 \mathrm{~d}\left(2 \mathrm{H},{ }^{3} \mathrm{~J}=7.3 \mathrm{~Hz}\right), 6.82 \mathrm{~s}(2 \mathrm{H}), 6.90 \mathrm{t}\left(2 \mathrm{H},{ }^{3} \mathrm{~J}=7.8 \mathrm{~Hz}\right), 7.02$ s $(2 \mathrm{H}), 7.19 \mathrm{t}\left(2 \mathrm{H},{ }^{3} J=7.5 \mathrm{~Hz}\right), 7.21 \mathrm{t}\left(2 \mathrm{H},{ }^{3} J=7.7 \mathrm{~Hz}\right), 7.32-7.52$ $\mathrm{m}(11 \mathrm{H}), 7.89-8.10 \mathrm{~m}(8 \mathrm{H})\left(\mathrm{NH}\right.$ protons were not assigned). ${ }^{13} \mathrm{C}$ NMR $\left(\mathrm{CDCl}_{3}, 298 \mathrm{~K}\right) \delta_{\mathrm{C}}$ ppm: 14.04 (2C), 14.18 (6C), 15.45 (4C), 15.52 (4C), 22.84 (8C), 26.81 (8C), 27.55 (2C), 28.26 (1C), 28.74 (1C), $32.52(2 \mathrm{C}), 32.59$ (6C), $33.10(8 \mathrm{C}), 42.61(1 \mathrm{C}), 47.66(1 \mathrm{C})$, 52.79 (1C), 53.40 (1C), 68.73 (1C), 70.55 (4C), 70.78 (2C), 70.94 (4C), 71.12 (1C), 97.45 (4C), 110.53, 110.81, 111.20, 112.31, 115.31, $115.98,116.41,120.19-120.47 \mathrm{~m}, 129.24,129.34,129.49,133.31$, $134.02,134.11,134.28,138.13,142.63,143.01,143.34-143.46 \mathrm{~m}$, $144.82,146.38,147.71,148.18,148.88,149.21$ (aromatic carbon atoms are not enough characteristic as they possess very close chemical shifts and form complicated multiplets). 
Pentamacrocycle $\boldsymbol{8}$ was isolated as the fourth compound in the synthesis of trismacrocycle 5. Eluent: $\mathrm{CH}_{2} \mathrm{Cl}_{2}-\mathrm{MeOH}$ 100:1. Yield $13 \mathrm{mg}(9 \%)$, dark-red solid. $\mathrm{m} / \mathrm{z}$ (MALDI-TOF) found: 2823.64. $\mathrm{C}_{178} \mathrm{H}_{218} \mathrm{~N}_{14} \mathrm{O}_{9} \mathrm{Zn}_{2}$ requires $2823.56[\mathrm{M}]^{+}$. ${ }^{1} \mathrm{H} \mathrm{NMR}$ $\left(\mathrm{CDCl}_{3}, 298 \mathrm{~K}\right) \delta_{\mathrm{H}}$ ppm: $1.00 \mathrm{t}\left(24 \mathrm{H},{ }^{3} J=7.1 \mathrm{~Hz}\right), 1.51-1.65 \mathrm{~m}$ $(20 \mathrm{H}), 1.72-1.83 \mathrm{~m}(16 \mathrm{H}), 2.17$ br.s $(8 \mathrm{H}), 2.13-2.28 \mathrm{~m}(16 \mathrm{H}), 3.07$ br.s $(4 \mathrm{H}), 3.30-3.48 \mathrm{~m}(24 \mathrm{H}), 3.57$ br.t $\left(4 \mathrm{H},{ }^{3} \mathrm{~J}_{o b s}=4.4 \mathrm{~Hz}\right), 3.64 \mathrm{t}$ $\left(8 \mathrm{H},{ }^{3} J=5.4 \mathrm{~Hz}\right), 4.00$ br.s $(16 \mathrm{H}), 4.20$ br.s $(8 \mathrm{H}), 5.80 \mathrm{~s}(2 \mathrm{H}), 6.06$ $\mathrm{s}(1 \mathrm{H}), 6.07 \mathrm{~s}(1 \mathrm{H}), 6.33 \mathrm{~s}(2 \mathrm{H}), 6.87 \mathrm{~d}\left(2 \mathrm{H},{ }^{3} \mathrm{~J}=8.5 \mathrm{~Hz}\right), 7.05 \mathrm{t}$ $\left(2 \mathrm{H},{ }^{3} J=7.5 \mathrm{~Hz}\right), 7.13 \mathrm{~d}\left(2 \mathrm{H},{ }^{3} J=7.6 \mathrm{~Hz}\right), 7.27-7.52 \mathrm{~m}(20 \mathrm{H}), 7.86$ d $\left(4 \mathrm{H},{ }^{3} J=7.6 \mathrm{~Hz}\right), 7.97 \mathrm{~d}\left(4 \mathrm{H},{ }^{3} J=7.5 \mathrm{~Hz}\right), 10.15 \mathrm{~s}(4 \mathrm{H})(\mathrm{NH}$ were not assigned). ${ }^{13} \mathrm{C}$ NMR $\left(\mathrm{CDCl}_{3}, 298 \mathrm{~K}\right) \delta_{\mathrm{C}} \mathrm{ppm}$ : $14.22(8 \mathrm{C}), 15.41$ (4C), 15.51 (4C), 22.86 (8C), 26.88 (8C), 27.44 (2C), 27.87 (2C), 28.32 (2C), $32.66(8 \mathrm{C}), 33.25$ (8C), 42.96 (2C), 49.06 (2C), 49.46 (2C), 68.7 (2C), 70.3-70.9 m (16C), 97.14 (4C) 111.61, 111.88, $113.36,116.47,117.27,118.80,118.88,119.34,119.68,119.98$, $120.46,120.61,120.87,121.17,128.61,128.87,129.42,136.81$, $137.56,137.99,141.45,141.82,143.14,143.20,143.41,146.31$, $147.49,147.84,148.05,148.20,149.15$ (signals of aromatic carbon atoms are not enough characteristic due to line broadening of some signals and impossibility of the identification of some quaternary carbon atoms).

Tetramacrocyclic compound 16. Obtained from zinc porphyrinate 15 ( $0.3 \mathrm{mmol}, 226 \mathrm{mg})$, cryptand $12(0.15 \mathrm{mmol}, 92 \mathrm{mg})$ in the presence of $\mathrm{Pd}(\mathrm{dba})_{2}(16 \mathrm{~mol} \%, 14 \mathrm{mg})$, BINAP (18 mol\%, $17 \mathrm{mg}), t \mathrm{BuONa}(0.45 \mathrm{mmol}, 43 \mathrm{mg})$ in $5 \mathrm{ml}$ of dioxane. Eluent: $\mathrm{CH}_{2} \mathrm{Cl}_{2}-\mathrm{MeOH}$ 10:1. Yield $68 \mathrm{mg}$ (23\%), dark-red solid. $\mathrm{m} / \mathrm{z}$ (MALDI-TOF) found: 1955.05. $\mathrm{C}_{118} \mathrm{H}_{146} \mathrm{~N}_{12} \mathrm{O}_{6} \mathrm{Zn}_{2}$ requires 1955.01 $[\mathrm{M}]^{+} .{ }^{1} \mathrm{H}$ NMR $\left(\mathrm{CDCl}_{3}, 298 \mathrm{~K}\right) \delta_{\mathrm{H}} \mathrm{ppm}: 0.97 \mathrm{t}\left(12 \mathrm{H},{ }^{3} \mathrm{~J}=6.8 \mathrm{~Hz}\right)$, 1.49 sextet $\left(8 \mathrm{H},{ }^{3} \mathrm{~J}=7.0 \mathrm{~Hz}\right), 1.63$ quintet $\left(8 \mathrm{H},{ }^{3} \mathrm{~J}=6.2 \mathrm{~Hz}\right), 2.01-2.14$ m (12H), 2.34 br.s $(8 \mathrm{H}), 2.56 \mathrm{~s}(12 \mathrm{H}), 3.15-3.46 \mathrm{~m}(24 \mathrm{H}), 3.31$ $\mathrm{s}(12 \mathrm{H}), 3.36 \mathrm{~s}(12 \mathrm{H}), 3.47-3.65 \mathrm{~m}(16 \mathrm{H}), 6.76-6.80 \mathrm{~m}(4 \mathrm{H})$, 7.30-7.85 m (10H), 7.90-7.96 m (4H).

Tetramacrocyclic compound 17. Obtained from zinc porphyrinate $15(0.348 \mathrm{mmol}, 262 \mathrm{mg})$, cryptand $13(0.174 \mathrm{mmol}$, $115 \mathrm{mg})$ in the presence of $\operatorname{Pd}(\mathrm{dba})_{2}(16 \mathrm{~mol} \%, 16 \mathrm{mg})$, DavePhos $(18 \mathrm{~mol} \%, 12 \mathrm{mg}), t \mathrm{BuONa}(0.522 \mathrm{mmol}, 50 \mathrm{mg})$ in $3.5 \mathrm{ml}$ of dioxane. Eluent: $\mathrm{CH}_{2} \mathrm{Cl}_{2}-\mathrm{MeOH} 10: 1$. Yield $70 \mathrm{mg}(20 \%)$, dark-red crystalline powder, m.p. $145-150^{\circ} \mathrm{C} . \mathrm{m} / \mathrm{z}$ (MALDI-TOF) found: 1998.99. $\mathrm{C}_{120} \mathrm{H}_{150} \mathrm{~N}_{12} \mathrm{O}_{7} \mathrm{Zn}_{2}$ requires $1999.03[\mathrm{M}]^{+}$. UV-Vis $\left(\mathrm{CH}_{3} \mathrm{CN}\right) \lambda_{\max } \mathrm{nm}(\lg \varepsilon): 409$ (5.49). ${ }^{1} \mathrm{H} \mathrm{NMR}\left(\mathrm{CDCl}_{3}, 298 \mathrm{~K}\right) \delta_{\mathrm{H}}$ ppm: $0.97 \mathrm{t}\left(12 \mathrm{H},{ }^{3} J=7.2 \mathrm{~Hz}\right), 1.52$ sextet $\left(8 \mathrm{H},{ }^{3} J=7.1 \mathrm{~Hz}\right), 1.67$ quintet $\left(8 \mathrm{H},{ }^{3} \mathrm{~J}=6.9 \mathrm{~Hz}\right), 2.11$ quintet $\left(8 \mathrm{H},{ }^{3} \mathrm{~J}=6.6 \mathrm{~Hz}\right), 2.19$ quintet $\left(4 \mathrm{H},{ }^{3} J=6.4 \mathrm{~Hz}\right), 2.34$ br.s $(8 \mathrm{H}), 2.50 \mathrm{~s}(12 \mathrm{H}), 2.87$ br.s $(8 \mathrm{H}), 3.00$ br.s $(8 \mathrm{H}), 3.23-3.51 \mathrm{~m}(12 \mathrm{H}), 3.35 \mathrm{~s}(24 \mathrm{H}), 3.69$ br.s $(4 \mathrm{H}), 3.79$ br.s $(4 \mathrm{H}), 3.86 \mathrm{t}\left(4 \mathrm{H},{ }^{3} J=7.6 \mathrm{~Hz}\right), 6.70$ br.s $(4 \mathrm{H}), 6.96$ br.s $(4 \mathrm{H})$, $7.32-7.40 \mathrm{~m}(4 \mathrm{H}), 7.47-7.57 \mathrm{~m}(4 \mathrm{H}), 9.35 \mathrm{~s}(2 \mathrm{H}), 9.70 \mathrm{~s}(4 \mathrm{H}) .{ }^{13} \mathrm{C}$ $\mathrm{NMR}\left(\mathrm{CDCl}_{3}, 298 \mathrm{~K}\right) \delta_{\mathrm{C}} \mathrm{ppm}$ : $11.4(4 \mathrm{C}), 12.0(4 \mathrm{C}), 14.1(4 \mathrm{C}), 15.3$ (4C), 22.7 (4C), 26.3 (4C), 27.8 (2C), 32.3 (4C), 32.9 (4C), 49.1 (2C), 53.2 (4C), 58.4 (2C), 68.0 (2C), 68.2 (4C), 69.1 (4C), 69.6 (2C), 70.0 (2C), 95.8 (2C), $96.6(4 \mathrm{C}), 113.3,119.6,121.7,125.5$, $126.0,127.8,129.9,135.4,137.7,140.8,145.0,146.6,147.4,147.6$ (6 quaternary carbon atoms were not assigned, not all quaternary carbon atoms of the porphyrin moiety were integrated).

Tetramacrocyclic compound 19. Obtained from zinc porphyrinate $18(0.316 \mathrm{mmol}, 238 \mathrm{mg})$, cryptand $14(0.158 \mathrm{mmol}, 100 \mathrm{mg})$ in the presence of Pd(dba) $2(16 \mathrm{~mol} \%, 15 \mathrm{mg})$, DavePhos (18 $\mathrm{mol} \%$, $11 \mathrm{mg}), t \mathrm{BuONa}(0.5 \mathrm{mmol}, 48 \mathrm{mg})$ in $2 \mathrm{ml}$ of dioxane. Eluent: $\mathrm{CH}_{2} \mathrm{Cl}_{2}-\mathrm{MeOH} 10: 1$. Yield $33 \mathrm{mg}$ (11 \%), dark-red solid. $\mathrm{m} / \mathrm{z}$ (MALDI-TOF) found: 1971.03. $\mathrm{C}_{118} \mathrm{H}_{146} \mathrm{~N}_{12} \mathrm{O}_{7} \mathrm{Zn}_{2}$ requires 1971.00 $[\mathrm{M}]^{+} .{ }^{1} \mathrm{H}$ NMR $\left(\mathrm{CDCl}_{3}, 298 \mathrm{~K}\right) \delta_{\mathrm{H}} \mathrm{ppm}: 0.99 \mathrm{t}\left(12 \mathrm{H},{ }^{3} \mathrm{~J}=7.1 \mathrm{~Hz}\right), 1.52$ br.sextet $\left(8 \mathrm{H},{ }^{3} \mathrm{~J}=6.0 \mathrm{~Hz}\right), 1.65$ br.s $(8 \mathrm{H}), 2.04$ br.s $(8 \mathrm{H}), 2.37$ br.s $(8 \mathrm{H}), 2.60 \mathrm{~s}(12 \mathrm{H}), 3.08$ br.s $(8 \mathrm{H}), 3.27-3.60 \mathrm{~m}(24 \mathrm{H}), 3.33 \mathrm{~s}(12 \mathrm{H})$, $3.35 \mathrm{~s}(12 \mathrm{H}), 3.66$ br.s $(8 \mathrm{H}), 3.78$ br.s $(4 \mathrm{H}), 6.46$ br.s $(2 \mathrm{H}), 6.85$ br.s $(2 \mathrm{H}), 6.93$ br.s $(2 \mathrm{H}), 7.13$ br.s $(2 \mathrm{H}), 7.29$ br.d $\left(2 \mathrm{H},{ }^{3} \mathrm{~J}_{\mathrm{obs}}=7.8 \mathrm{~Hz}\right)$, 7.41 br.s (2H), 7.54-7.62 m (2H), 7.71 br.d $\left(2 \mathrm{H},{ }^{3} J_{o b s}=7.5 \mathrm{~Hz}\right), 9.65$ $\mathrm{s}(4 \mathrm{H}), 9.77 \mathrm{~s}(2 \mathrm{H}),{ }^{13} \mathrm{C} \mathrm{NMR}\left(\mathrm{CDCl}_{3}, 298 \mathrm{~K}\right) \delta_{\mathrm{C}}{ }_{\mathrm{ppm}} 11.3(4 \mathrm{C})$, 12.0 (4C), 14.1 (4C), 15.3 (4C), 22.7 (4C), 26.1 (4C), 32.3 (4C), 32.9
(4C), 51.9 (2C), 52.5 (4C), 58.2 (2C), 67.9-70.6 m (14C), $95.6(2 \mathrm{C})$, 96.6 (4C), 119.1, 120.2, 121.3, 122.2, 126.7, 127.8, 129.0, 135.3, $137.3,137.6,140.7,145.0,146.3,146.5,146.7,147.4,147.7,148.0$ (6 quaternary carbon atoms were not assigned, not all quaternary carbon atoms of the porphyrin moiety were integrated).

Trismacrocycle 20 was isolated as the second compound in the synthesis of tetramacrocycle 19. Eluent: $\mathrm{CH}_{2} \mathrm{Cl}_{2}-\mathrm{MeOH} 5: 1$. Yield $33 \mathrm{mg}$ (16\%), dark-red crystalline powder. M.p. 147-152 ${ }^{\circ} \mathrm{C} . \mathrm{m} / z$ (MALDI-TOF) found: $1300.6934 . \mathrm{C}_{76} \mathrm{H}_{100} \mathrm{~N}_{8} \mathrm{O}_{7} \mathrm{Zn}$ requires $1300.7006[\mathrm{M}]^{+}$. UV-Vis $\left(\mathrm{CH}_{3} \mathrm{CN}\right) \lambda_{\max }(\lg \varepsilon) \mathrm{nm}: 411(5.32) .{ }^{1} \mathrm{H}$ NMR $\left(\mathrm{CDCl}_{3}, 298 \mathrm{~K}\right) \delta_{\mathrm{H}}$ ppm: $0.98 \mathrm{t}\left(6 \mathrm{H},{ }^{3} J=7.3 \mathrm{~Hz}\right), 1.55$ sextet $\left(4 \mathrm{H},{ }^{3} J=7.4 \mathrm{~Hz}\right), 1.72$ quintet $\left(4 \mathrm{H},{ }^{3} J=7.4 \mathrm{~Hz}\right), 2.23$ quintet $(4 \mathrm{H}$, $\left.{ }^{3} J=7.4 \mathrm{~Hz}\right), 2.40$ br.s $(8 \mathrm{H}), 2.61 \mathrm{~s}(6 \mathrm{H}), 2.90$ br.s $(8 \mathrm{H}), 3.08$ br.s $(8 \mathrm{H}), 3.15$ br.s $(4 \mathrm{H}), 3.33$ br.s $(4 \mathrm{H}), 3.35 \mathrm{~s}(4 \mathrm{H}), 3.45 \mathrm{~s}(6 \mathrm{H}), 3.49 \mathrm{~s}$ (6H), 3.62 br.t $\left(4 \mathrm{H},{ }^{3} J=4.6 \mathrm{~Hz}\right), 3.89 \mathrm{t}\left(4 \mathrm{H},{ }^{3} J=5.6 \mathrm{~Hz}\right), 6.49$ br.s $(2 \mathrm{H}), 6.79$ br.s $(2 \mathrm{H}), 7.16$ br.s $(4 \mathrm{H}), 7.28 \mathrm{~d}\left(1 \mathrm{H},{ }^{3} \mathrm{~J}=7.7 \mathrm{~Hz}\right), 7.56 \mathrm{t}$ $\left(1 \mathrm{H},{ }^{3} J=7.6 \mathrm{~Hz}\right), 7.64 \mathrm{~s}(1 \mathrm{H}), 7.74 \mathrm{~d}\left(1 \mathrm{H},{ }^{3} J=6.9 \mathrm{~Hz}\right), 9.70 \mathrm{~s}(1 \mathrm{H})$, $9.84 \mathrm{~s}(2 \mathrm{H})(\mathrm{NH}$ proton was not assigned).

\section{Results and Discussion}

At the first step of our research we synthesized the macrocycle 3 comprising 3,3'-disubstituted biphenyl and trioxadiamine moieties. The synthesis was carried out according to a known procedure, ${ }^{[24]}$ starting from 3,3'-dibromobiphenyl (1) and linear trioxadiamine 2, in the presence of a standard catalytic system $\mathrm{Pd}(\mathrm{dba})_{2} /$ BINAP $\quad\left(\mathrm{dba}=\right.$ dibenzylideneacetone, $\quad \mathrm{BINAP}=2,2{ }^{2}$ bis(diphenylphosphino)-1,1'-binaphthalene). The target compound was isolated in $40 \%$ yield and introduced in the second catalytic reaction with zinc 5,15-di(4bromophenyl)porphyrinate 4 (Scheme 1). This coupling was conducted using DavePhos phosphine ligand (DavePhos=2-(dicyclohexylphosphino)-2,2'-dimethylaminobiphenyl) as special experiment revealed the superiority of this donor ligand over more conventional BINAP in the arylation of the secondary amino groups with bromophenyl porphyrinates. It was also important to use $\mathrm{Zn}(\mathrm{II})$ complex as free porphyrins often failed to give desired products in similar arylation reactions. The ratio of the starting compounds 3:4 was taken as 2:1, as a result we obtained a series of polymacrocycles: the expected trimacrocycle $\mathbf{5}$, i.e. the product of the diamination of di(bromophenyl) porphyrin, the product of monoamination $\mathbf{6}$, and oligomeric tetracyclic and pentacyclic compounds 7 and $\mathbf{8}$ (Scheme 1). Compounds $\mathbf{6}$ and 7 were formed in the result of the catalytic amination and catalytic reduction of the $\mathrm{C}-\mathrm{Br}$ bonds. The isolated yields of all these compounds were small and ranged from 9 to $16 \%$. The isolation of individual products by chromatography on silica gel was quite tedious and obviously oligomers with higher molecular masses were not obtained in individual state.

Another structural type of porphyrin-containing polymacrocycles is based on the cryptand-like derivatives of diazacrown ethers 12-14. These compounds were obtained by a previously described method ${ }^{[25]}$ in sufficient yields (24-38\%) (Scheme 2), they differ by the size of the central diazacrown moiety as well by the nature of the trioxadiamine linker and substitution pattern in the benzyl spacers. Cryptands 12 and $\mathbf{1 3}$ were introduced in the $\operatorname{Pd}(0)$ catalyzed diarylation reaction with zinc 5-(4-bromophenyl) porphyrinate 15 (2 equiv.) using the same catalytic system 


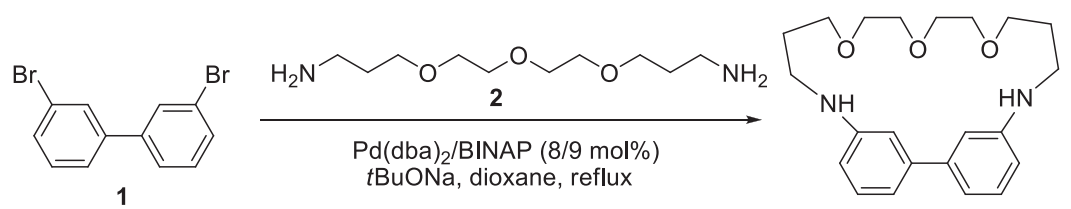

3, $40 \%$

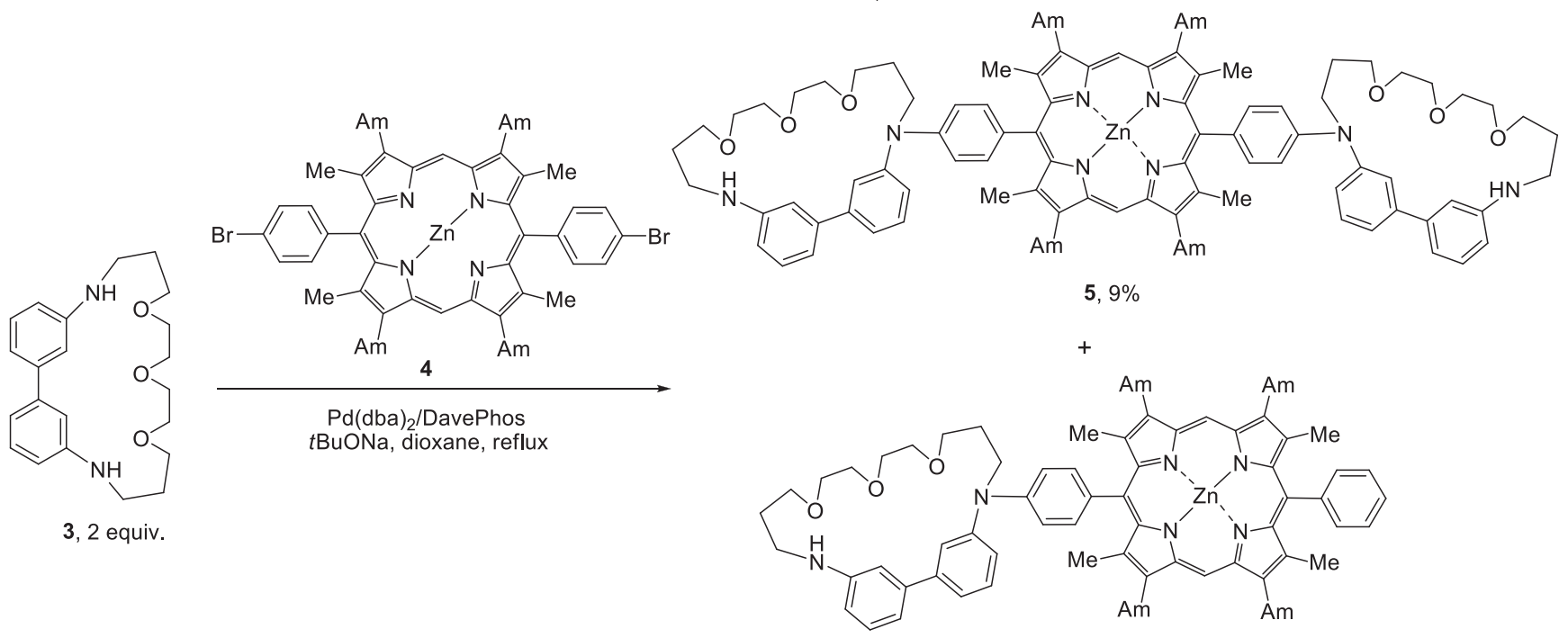

$6,16 \%$
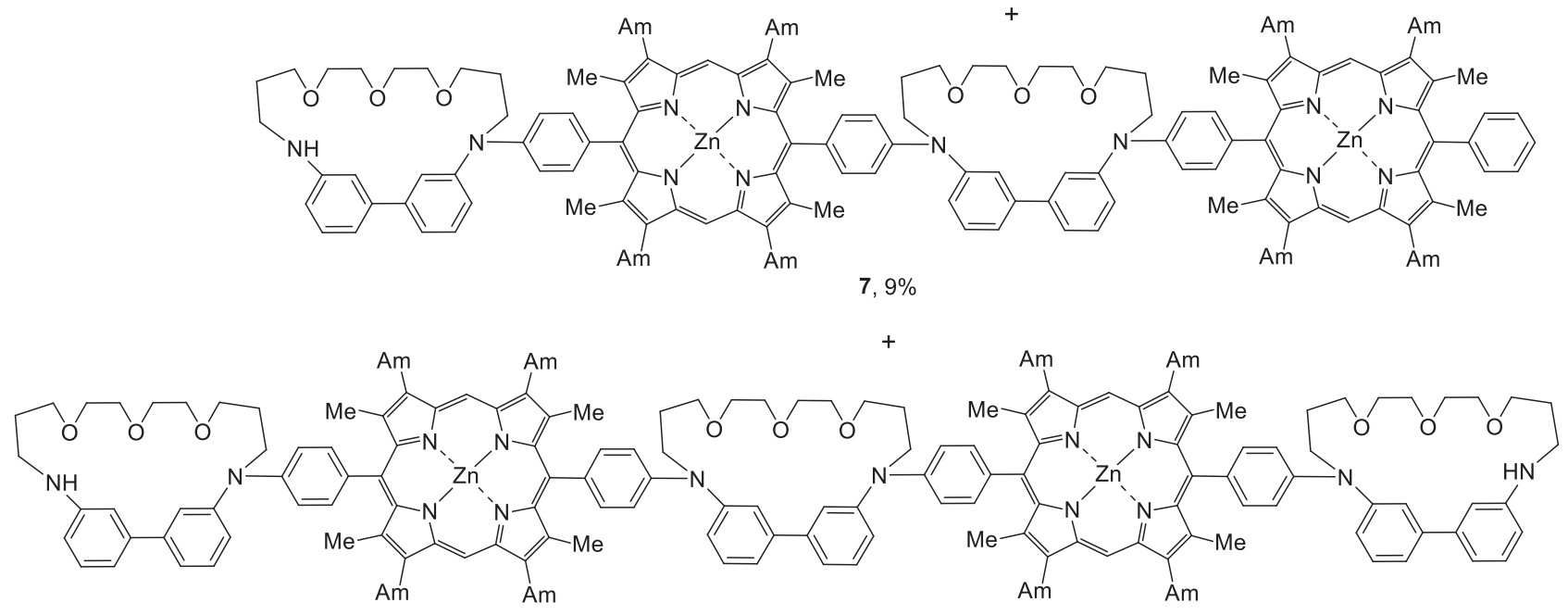

$8,9 \%$

Scheme 1.

$\mathrm{Pd}(\mathrm{dba})_{2} /$ DavePhos as in the previous case. As the number of reaction centers decreased in these processes, the target tetramacrocyclic products of diarylation $\mathbf{1 6}$ and $\mathbf{1 7}$ were isolated in fairly better yields (23 and $20 \%$, respectively) (Scheme 2). It is interesting that in the case of the reaction of zinc porphyrinate $\mathbf{1 5}$ with the cryptand $\mathbf{1 2}$ BINAP was found to be also efficient for the coupling.

Next we explored the possibility to introduce an isomeric zinc 5-(3-bromophenyl)porphyrinate $\mathbf{1 8}$ in the similar reaction with the cryptand $\mathbf{1 4}$ (Scheme 3). Porphyrin derivative $\mathbf{1 8}$ possesses less active bromine atom compared to its isomer $\mathbf{1 5}$ in which bromine is situated in para-position to a strong electron-withdrawing porphyrin unit. As expected, the reaction with less reactive zinc porphyrinate $\mathbf{1 8}$ resulted in a lower yield of the desired bisporphyrin tetramacrocycle $19(11 \%)$ while the product of monoarylation, i.e. trimacrocycle $\mathbf{2 0}$ was obtained in $16 \%$ yield (Scheme 3 ).

We investigated the possibilities of polymacrocyclic compounds to act as fluorescent chemosensors for metal cations. In the course of investigation UV-Vis and fluorescent spectra of the polymacrocycles 5, 6, 17 and 20 were recorded in $\mathrm{MeCN}$ in the presence of 1, 2, 5 equiv. (in some cases also 10, 20, 30 equiv.) of corresponding metal perchlorates: $\mathrm{Li}(\mathrm{I}), \mathrm{Na}(\mathrm{I}), \mathrm{K}(\mathrm{I}), \mathrm{Mg}(\mathrm{II}), \mathrm{Ca}(\mathrm{II}), \mathrm{Ba}(\mathrm{II})$, $\mathrm{Al}(\mathrm{III}), \mathrm{Mn}(\mathrm{II}), \mathrm{Fe}(\mathrm{II}), \mathrm{Co}(\mathrm{II}), \mathrm{Ni}(\mathrm{II}), \mathrm{Cr}(\mathrm{III}), \mathrm{Cu}(\mathrm{II})$, $\mathrm{Zn}(\mathrm{II}), \mathrm{Cd}(\mathrm{II}), \mathrm{Hg}(\mathrm{II}), \mathrm{Ag}(\mathrm{I}), \mathrm{Pb}(\mathrm{II})$. It was found out that the bismacrocyclic compound $\mathbf{6}$ could serve as the fluorescent molecular probe for $\mathrm{Cu}(\mathrm{II}), \mathrm{Al}(\mathrm{III})$ and $\mathrm{Cr}(\mathrm{III})$ cations because the addition of only these metals led to full quenching of emission (Figure S1). To achieve this full quenching, one needs 2 equiv. of Al(III) (Figure S2), 5 equiv. 
<smiles>Brc1ccc(CN2CCOCCOCCN(Cc3ccc(Br)cc3)CCOCC2)cc1</smiles>

9: $\mathrm{n}=1, m-\mathrm{Br}$ 10: $\mathrm{n}=2, m-\mathrm{Br}$
$11: \mathrm{n}=2, \mathrm{Br}$
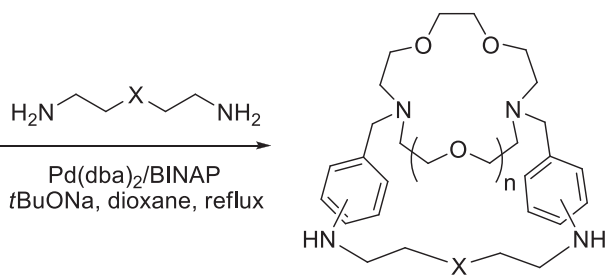

12: $\mathrm{n}=1, m-\mathrm{NH}, \mathrm{X}=\mathrm{CH}_{2} \mathrm{OCH}_{2} \mathrm{CH}_{2} \mathrm{OCH}_{2} \mathrm{CH}_{2} \mathrm{OCH}_{2}, 38 \%$ 13: $\mathrm{n}=2, m-\mathrm{NH}, \mathrm{X}=\mathrm{CH}_{2} \mathrm{OCH}_{2} \mathrm{CH}_{2} \mathrm{OCH}_{2} \mathrm{CH}_{2} \mathrm{OCH}_{2}, 36 \%$ 14: $\mathrm{n}=2, p-\mathrm{NH}, \mathrm{X}=\mathrm{OCH}_{2} \mathrm{CH}_{2} \mathrm{OCH}_{2} \mathrm{CH}_{2} \mathrm{O}, 24 \%$
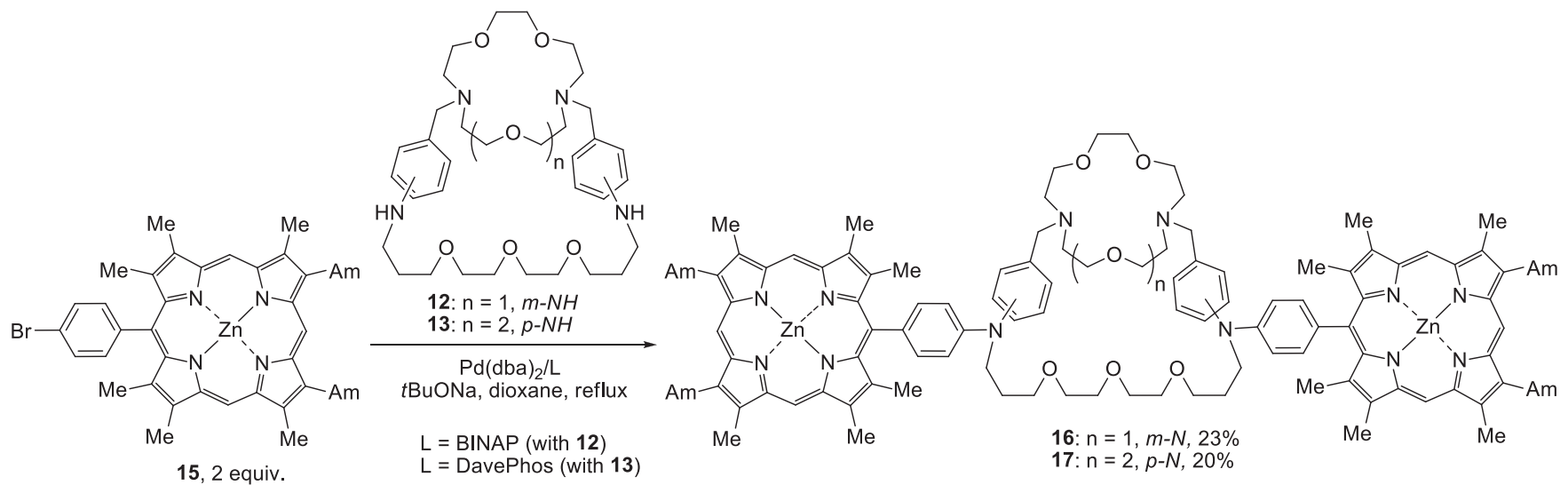

Scheme 2.
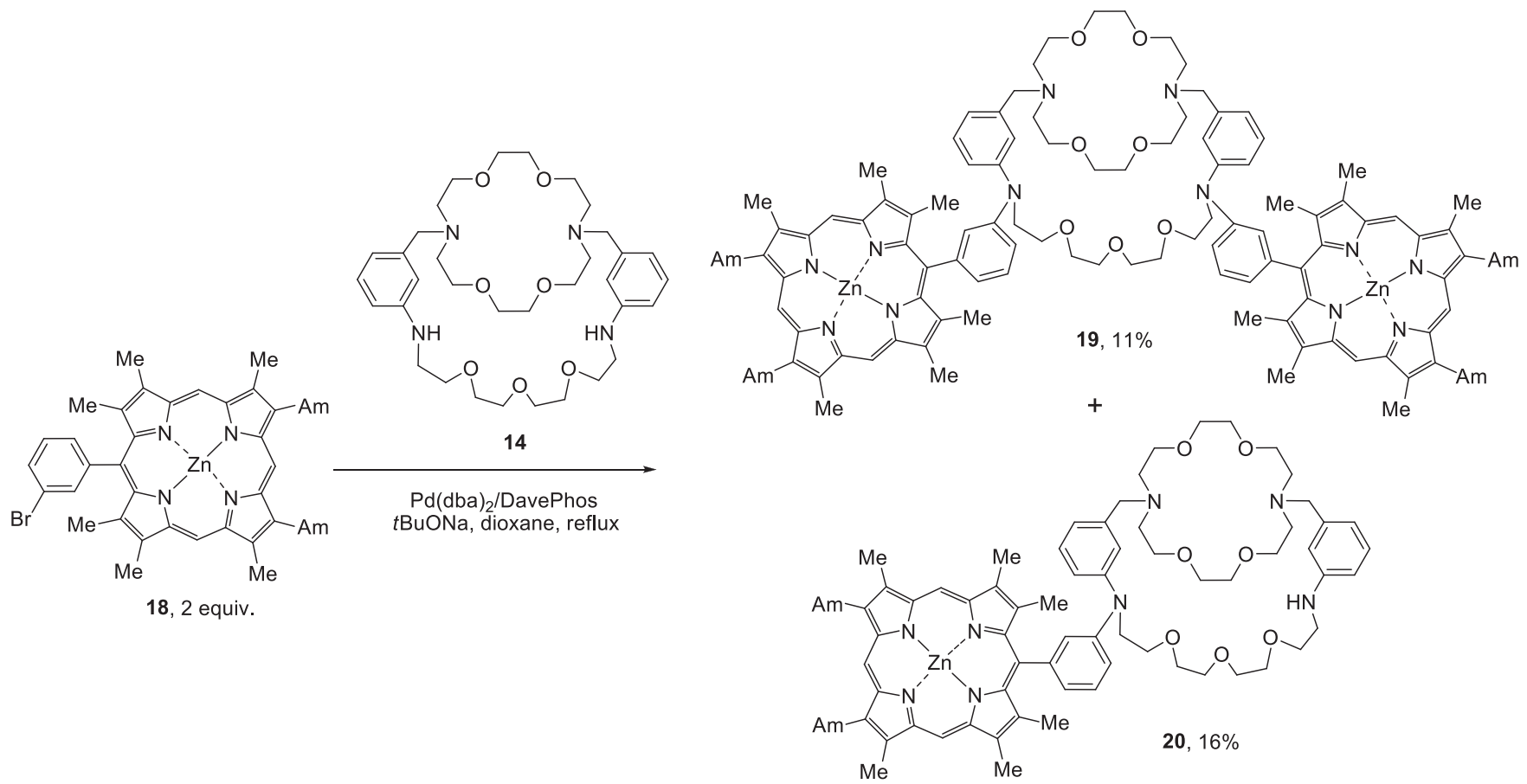

Scheme 3.

of $\mathrm{Cr}$ (III) (Figure S3) or only 1 equiv. of $\mathrm{Cu}$ (II). We carried out both UV-Vis and fluorescent titrations (Figures S4, S5) and calculated the stability constants of two complexes with $\mathrm{Cr}$ (III): for (6) $\mathrm{Cr}$ (III) complex $\lg K=6.20 \pm 0.10$ and for (6) $\cdot 2 \mathrm{Cr}$ (III) $\lg K=12.25 \pm 0.07$. In UV-Vis spectra $\mathrm{Cr}$ (III) and $\mathrm{Al}(\mathrm{III})$ caused insignificant decrease in the intensity of the absorption band with bathochromic shift by $12 \mathrm{~nm}$ while the addition of $\mathrm{Cu}$ (II) salt led to a disappearance of this absorption band.
Emission of the trismacrocycle $\mathbf{5}$ with one central porphyrin and two peripherical diazatrioxamacrocycles is less susceptible to the addition of $\mathrm{Cr}$ (III) as only 10 equiv. led to full quenching (Figure S6). However, $\mathrm{Cu}$ (II) and Al(III) quench emission efficiently (Figure S7), thus this molecule can be also regarded as a molecular probe for these three cations. The effect of the metal cations on the UV-Vis spectra are quite similar to that of compound 6 (Figure S8). 
Tetramacrocyclic ligand $\mathbf{1 7}$ can be seen as a chemosensor for $\mathrm{Cu}$ (II) cations as only this metal fully quenches its emission upon addition of 10 equiv. (Figures S9, S10). Moreover, this detector is characterized by a low detection limit $(0.23 \mu \mathrm{M})$. The stability constant of the complex (17) $\mathrm{Cu}$ (II) was found to be equal $\lg K=5.15 \pm 0.04$ by fluorimetric titration. Trismacrocyclic compound $\mathbf{2 0}$ which includes only one porphyrin structural unit and possesses a slightly shorter trioxadiamine linker though selectively responses for $\mathrm{Cu}(\mathrm{II})$ cations, is less efficient in detecting this metal as its emission diminishes only 2.5 times after the addition of 15 equiv. of this metal (Figures S11, S12). This fact implies the necessity of fine tuning of the polymacrocyclic structures for increasing selectivity and sensitivity of the detector. In UV-Vis spectra of polymacrocycles 17 and 20 the decrease in the intensity of the absorption maxima upon addition of $\mathrm{Cu}(\mathrm{II})$ cations without notable shift was observed (Figures S13, S14).

\section{Conclusions}

To sum up, our research revealed the possibility to construct polymacrocyclic compounds incorporating porphyrin and oxaazamacrocyclic structural units using $\operatorname{Pd}(0)$-catalyzed amination reactions. They were tested as potential fluorimetric detectors of metal cations and the strong dependence of the emission quenching in the presence of certain cations on the type and number of macrocycles attached to porphyrin units was firmly established. Two of them (monoporphyrin-based bisand trismacrocycles $\mathbf{5}$ and $\mathbf{6}$ ) were found to be prospective fluorescent detectors for $\mathrm{Al}(\mathrm{III}), \mathrm{Cr}$ (III) and $\mathrm{Cu}(\mathrm{II})$ while tetramacrocyclic bisporphyrin derivative $\mathbf{1 7}$ can serve as the fluorimetric chemosensor for $\mathrm{Cu}$ (II).

Acknowledgements. The authors acknowledge the financial support of the RFBR grant 16-29-10685 for the synthesis of starting compounds and spectral investigations and RSF grant 14-23-00186P for the catalytic synthesis of porphyrincontaining polymacrocycles.

\section{References}

1. Lu X.Q., Guo Y., Chen Q.Y. Synlett 2011, 2011(1), 77-80.

2. Cheng F.Y., Zhang S., Adronov A., Echegoyen L., Diederich F. Chem. Eur. J. 2006, 12, 6062-6070.
3. Filatov M.A., Guilard R., Harvey P.D. Org. Lett. 2010, 12, 196-199.

4. Hyslop A.G., Kellett M.A., Iovine P.M., Therien M.J. J. Am. Chem. Soc. 1998, 120, 12676-12677.

5. Chung L.L., Chang C.J., Nocera D.G. J. Org. Chem. 2003, 68, 4075-4078.

6. Yu L.H., Lindsey J.S. Tetrahedron 2001, 57, 9285-9298.

7. Sergeeva N.N., Scala A., Bakar M.A., O'Riordan G., O'Brien J., Grassi G., Senge M.O. J. Org. Chem. 2009, 74, 7140-7147.

8. Frampton M.J., Akdas H., Cowley A.R., Rogers J.E., Slagle J.E., Fleitz P.A., Drobizhev M., Rebane A., Anderson H.L. Org. Lett. 2005, 7, 5365-5368.

9. Odobel F., Suresh S., Blart E., Nicolas Y., Quintard J.P., Janvier P., Le Questel J.Y., Illien B., Rondeau D., Richomme P., Haupl T., Wallin S., Hammarstorm L. Chem. Eur. J. 2002, 8, 3027-3046.

10. Fazekas M., Pintea M., Senge M.O., Zawadzka M. Tetrahedron Lett. 2008, 49, 2236-2239.

11. Sato T., Nakagawa T., Okada H., Matsuo Y. J. Porphyrins Phthalocyanines 2015, 19, 451-458.

12. Severac M., Le Pleux L., Scarpaci A., Blart E., Odobel F. Tetrahedron Lett. 2007, 48, 6518-6522.

13. Polevaya Y.P., Tyurin V.S., Beletskaya I.P. J. Porphyrins Phthalocyanines 2014, 18, 20-34.

14. Yaschuk Y.P., Tyurin V.S., Beletskaya I.P. Macroheterocycles 2012, 5, 302-307.

15. Esdaile L.J., Senge M.O., Arnold D.P. Chem. Commun. 2006, 4192-4194.

16. Mikhalitsyna E.A., Tyurin V.S., Khrustalev V.N., Lonin I.S., Beletskaya I.P. Dalton Trans. 2014, 43, 3563-3575.

17. Mikhalitsyna E.A., Tyurin V.S., Beletskaya I.P. J. Porphyrins Phthalocyanines 2015, 19, 874-886.

18. Luo H.-Y., Jiang J.-H., Zhang X.-B., Li Ch.-Y., Shen G.-L., Yu R.-Q. Talanta 2007, 72, 575-581.

19. Li Ch.-Y., Xu F., Li Y.-E. Spectrochim. Acta, Part A 2010, 76, 197-201.

20. Han Z.-X., Luo H.-Y., Zhang X.-B., Kong R.-M., Shen G.-L., Yu R.-Q. Spectrochim. Acta, Part A 2009, 72, 1084-1088.

21. Yakushev A.A., Averin A.D., Maloshitskaya O.A., Syrbu S.A., Koifman O.I., Beletskaya I.P. Mendeleev Commun. 2016, 26, 199-201.

22. Yakushev A.A., Averin A.D., Maloshitskaya O.A., Syrbu S.A., Koifman O.I., Beletskaya I.P. Macroheterocycles 2016, 9, 65-72.

23. Ukai T., Kawazura H., Ishii Y., Bonnet J.J., Ibers J.A. J. Organomet. Chem. 1974, 65, 253-266.

24. Averin A.D., Uglov A.N., Buryak A.K., Beletskaya I.P. Mendeleev Commun. 2010, 20, 1-3.

25. Yakushev A.A., Chernichenko N.M., Anokhin M.V., Averin A.D., Buryak A.K., Denat F., Beletskaya I.P. Molecules 2014, 19, 940-965.

26. Mikhalitsyna E.A., Tyurin V.S., Nefedov S.E., Syrbu S.A., Semeikin A.S., Koifman O.I., Beletskaya I.P. Eur. J. Inorg. Chem. 2012, 36, 5979-5990. 